\title{
Viscosity of Aged Bio-Oils from Fast Pyrolysis of Beech Wood and Miscanthus: Shear Rate and Temperature Dependence
}

Junmeng Cai ${ }^{1,2, *}$, Scott W. Banks ${ }^{2}$, Yang Yang ${ }^{2}$, Surila Darbar ${ }^{2}$, Tony Bridgwater ${ }^{2}$

${ }^{1}$ Biomass Energy Engineering Research Center, Key Laboratory of Urban Agriculture (South)

Ministry of Agriculture, School of Agriculture and Biology, Shanghai Jiao Tong University, 800 Dongchuan Road, Shanghai 200240, People's Republic of China

${ }^{2}$ Bioenergy Research Group, European Bioenergy Research Institute (EBRI), Aston University, Aston Triangle, Birmingham B4 7ET, United Kingdom 


\section{ABSTRACT:}

The viscosity of four aged bio-oil samples was measured experimentally at various shear rates and temperatures by using a rotational viscometer. The experimental biooils were derived from fast pyrolysis of beech wood at 450,500 and $550{ }^{\circ} \mathrm{C}$, and Miscanthus at $500{ }^{\circ} \mathrm{C}$ (in this work, they were named as BW1, BW2, BW3 and MXG) in a bubbling fluidised bed reactor. The viscosity of all bio-oils kept constant at various shear rates at the same temperature, which indicated that they were Newtonian fluids. The viscosity of bio-oils was strongly dependent on the temperature, and by increasing the temperature from 30 to $80{ }^{\circ} \mathrm{C}$, the viscosity of BW1, BW2, BW3 and MXG decreased by $90.7 \%, 93.3 \%, 92.6 \%$ and $90.2 \%$, respectively. The Arrhenius viscosity model, which have been commonly used to represent the temperature dependence of the viscosity of many fluids, did not fit the viscosity-temperature experimental data of all bio-oils very well, especially in the low and high temperature regions. For comparison, the Williams-Landel-Ferry (WLF) model was also used. The results showed that the WLF model gave a very good description of the viscosity-temperature relationship of each bio-oil with very small residuals and the BW3 bio-oil had the strongest viscosity-temperature dependence.

KEY WORDS: bio-oil; viscosity; biomass; model; activation energy

\section{INTRODUCTION}

Fast pyrolysis is a technique of lignocellulosic biomass conversion into volatile 
form with the application of heat at a very high heating rate in an inert atmosphere. ${ }^{1}$ The volatiles produced can be converted into bio-oil by rapid condensing. There are many advantages of bio-oil over solid biomass, as bio-oil has a higher energy density and it is easier to transport and store. ${ }^{2}$

Viscosity is an important physical property of bio-oil that should be considered because it influences bio-oil's handling, mixing operation, pipeline flow, pumping, injection, atomisation, and combustion, etc.. ${ }^{2,3}$ Generally, high viscosity bio-oil may result in poor atomisation and incomplete combustion, coke deposition on injection nozzles and combustion chambers. ${ }^{4}$ The definition of the viscosity of a liquid is the measure of its resistance to gradual deformation by shear stress or tensile stress, which is originated from the internal structure and molecular interactions of the fluid. ${ }^{5}$ The variation of the viscosity with temperature is one of the most important parameters in the application of bio-oils, and the establishment of viscosity-temperature relationship can help us to understand the flow behaviors of bio-oil in a range of temperatures. ${ }^{6}$

In the literature, several models have been proposed for representing the variation of the viscosity of a fluid with the temperature. ${ }^{7}$ In 1886 , Reynolds proposed a simple model for the temperature-dependence of fluid viscosity. ${ }^{8}$

$$
\eta(T)=\eta_{R} \exp \left(-b_{R} T\right)
$$

where $\eta$ is the viscosity $(\mathrm{Pa} \cdot \mathrm{s}), T$ is the temperature $(\mathrm{K}), \eta_{R}(\mathrm{~Pa} \cdot \mathrm{s})$ and $b_{R}\left(\mathrm{~K}^{-1}\right)$ are the fitting parameters. The Reynolds model only works for a very limited range of temperatures. ${ }^{7}$ Therefore it will not be considered in this work.

The most commonly used model to correlate the viscosity-temperature 
relationship of a fluid is the Arrhenius model: ${ }^{7}$

$$
\eta(T)=\eta_{A} \exp \left(\frac{E_{A}}{R T}\right)
$$

where $R$ is the universal gas constant $\left(8.3145 \mathrm{~J} \cdot \mathrm{mol}^{-1} \cdot \mathrm{K}^{-1}\right), \quad \eta_{A}$ is the Arrhenius preexponential factor $(\mathrm{Pa} \cdot \mathrm{s})$ and $E_{A}$ is the Arrhenius activation energy $\left(\mathrm{J} \cdot \mathrm{mol}^{-1}\right)$. According to Equation (2), when $T \rightarrow \infty, \eta \rightarrow \eta_{A}$. So the physical meaning of $\eta_{A}$ is the infinite-temperature viscosity. The Arrhenius model has usually been used to describe the viscosity-temperature relationship of bio-oils in the literature. ${ }^{9-12}$ However, according to our results, it is unsuitable for describing the low and high temperature regions of the viscosity-temperature profiles of bio-oil. Detailed information will be discussed in the section 'RESULTS AND DISCUSSION' of this work.

The Williams-Landel-Ferry (WLF) model has also been used extensively in the description of the dependence of the viscosity of a fluid with temperature: ${ }^{13}$

$$
\eta(T)=\eta_{\mathrm{wLF}} \exp \left[-\frac{C_{1}\left(T-T_{r}\right)}{C_{2}+\left(T-T_{r}\right)}\right]
$$

where $T_{r}$ is the reference temperature $(\mathrm{K}), \eta_{\mathrm{wLF}}(\mathrm{Pa} \cdot \mathrm{s}), C_{1}$ (dimensionless) and $C_{2}(\mathrm{~K})$ are empirical constants. In the original paper of Williams et al., ${ }^{13} T_{r}$ was predetermined, however, after Dobson, ${ }^{14}$ it has been taken as an adjustable parameter. The limitations of the WLF model of the viscosity-temperature relationship are that the parameters $C_{1}$ and $C_{2}$ in the WLF model are empirical parameters, and they have indeterminacy physical meaning. According to the literature, ${ }^{15}$ the higher the $C_{2} / C_{1}$ value, the lower the dependence of viscosity with temperature.

In the literature, ${ }^{16,17}$ some researchers correlated the fluid viscosity-temperature 
relationship using the equations in the following forms: $\eta=A+B T+C T^{2}$, $\eta=A+B T+C T^{2}+D T^{4} \quad, \quad \eta=\exp \left(A+\frac{B}{T+C}\right) \quad, \quad \eta=\exp \left(A+\frac{B}{T}+C T\right)$ $\eta=\exp \left(A+\frac{B}{T}+\frac{C}{T^{2}}\right)$ and $\eta=B+\frac{A-B}{1+\exp \left(\frac{T-C}{D}\right)} \quad$ where $A, B, C$ and $D$ are constants). In those equations, the parameters have no physical meaning. Therefore, it is difficult to establish the relationship between the parameter values of those equation and the characteristics of bio-oil.

Therefore, the aim of this work is to analyse the experimental viscosity data of four bio-oil samples from fast pyrolysis of beech wood and Miscanthus at various shear rates and temperatures using the Arrhenius and WLF models.

\section{MATERIALS AND EXPERIMENTS}

\subsection{Bio-Oil Sample Preparation}

Fast pyrolysis of beech wood and Miscanthus $\times$ giganteus were performed using a $1 \mathrm{~kg} \mathrm{~h}^{-1}$ continuous bubbling fluidised bed reactor. The reactor was fluidised with preheated nitrogen at three times the minimum fluidising velocity $\left(17 \mathrm{~L} \mathrm{~min}^{-1}\right)$. The reactor bed material was $1 \mathrm{~kg}$ of quartz sand with particle size between 600 and 710 $\mu \mathrm{m}$. Pyrolysis vapours passed through two heated cyclones in series to separate solid particles (char). Following the cyclones the vapours were condensed in a quench column. The aerosols were coalesced in a wet walled electrostatic precipitator (ESP). Following the ESP the gas passed through a water cooled condenser, two dry ice / acetone condensers in series and finally a cotton wool filter. Detailed information about 
the reactor and the pyrolysis process can be found in the literature. ${ }^{16}$

Four fast pyrolysis runs were completed using two different feedstocks at varied temperature (see Table 1). The collected pyrolysis liquid contained a considerable amount of the quench medium. Each bio-oil sample was centrifuged (30 min at 4000 $\mathrm{rpm})$ to remove all quench medium. Immediately after production, the bio-oil samples were stored in tightly sealed glass bottles in the dark at $4{ }^{\circ} \mathrm{C}$. The bio-oil samples have been stored for two years before physicochemical analyses and viscosity measurements presented in the next subsections.

Table 1. Raw materials and pyrolysis temperatures for bio-oil samples

\begin{tabular}{cccc}
\hline Bio-oil sample & Raw material & Sample size & Pyrolysis temperature $/{ }^{\circ} \mathrm{C}$ \\
\hline BW1 & Beech wood & $0.25-2.00 \mathrm{~mm}$ & 450 \\
BW2 & Beech wood & $0.25-2.00 \mathrm{~mm}$ & 500 \\
BW3 & Beech wood & $0.25-2.00 \mathrm{~mm}$ & 550 \\
MXG & Miscanthus $\times$ giganteus & $0.25-2.00 \mathrm{~mm}$ & 500 \\
\hline
\end{tabular}

\subsection{Elemental Analysis}

A Thermo Scientific Flash 2000 Elemental Analyser was used to determine the carbon, hydrogen, nitrogen, sulfur and oxygen contents of bio-oil samples. An average water content of each bio-oil sample was calculated from at least triplicate results.

\subsection{Heating value}


A Parr ${ }^{\mathrm{TM}} 6100$ Compensated Calorimeter was employed to measure the heating values of bio-oil samples. For each bio-oil sample, its heating value was measured three times and the average value was reported.

\subsection{Water Content}

The water content of each bio-oil sample was analysed by volumetric Karl-Fisher (KF) titration according to ASTM standard E203 - 08 using Hydranal ${ }^{\circledR}$ Medium K and Hydranal ${ }^{\circledR}$ Composite $5 \mathrm{~K}$. The titration was repeated three times and the average result was reported.

\subsection{Dynamic Viscosity Measurement}

According to Standards ASTM 7544-12 and ASTM D445-12, the viscosity of biooil is determined by measuring the time for a volume of bio-oil to flow under gravity through a glass capillary viscometer at a closely controlled and known temperature (usually $40^{\circ} \mathrm{C}$ ). However, the glass capillary viscometer can not be used to investigate the effect of the shear rate on the viscosity. Therefore, the dynamic viscosity of bio-oil was measured using a Brookfield Viscometer Model DV- II + Pro rotational viscometer with temperature control. It measures the torque required to rotate a spindle in bio-oil at a known speed. According to the operation instructions of the viscometer, to ensure the selection of the spindle rotational speed, the torque digital display reading should be in the range of $10-100 \%$.

Two viscosity test modes were used in this work: controlled shear rate (CSR) and 
viscosity-temperature (VT) modes. The shear rate is calculated based on the spindle surface area and the rotational speed. The spindle torque is measured for each shear rate value. Torque is converted into the rheological parameter shear stress using the shear area of the viscometer measuring system. The corresponding viscosity is calculated from the shear stress and shear rate values.

In the CSR mode, a spindle rotational speed - time profile is preset at a constant temperature. The diagram of the CSR mode is shown in Figure 1. In this work, a program was used to set an initial spindle rotational speed resulting in approximately a $10 \%$ torque digital display reading, then after every minute the speed was increased by $0.2 \mathrm{rpm}$ for 40 minutes. A temperature controlled water bath was used to maintain temperatures of $30,40,50,60,70$ and $80{ }^{\circ} \mathrm{C}$ with an accuracy of $\pm 0.1^{\circ} \mathrm{C}$.

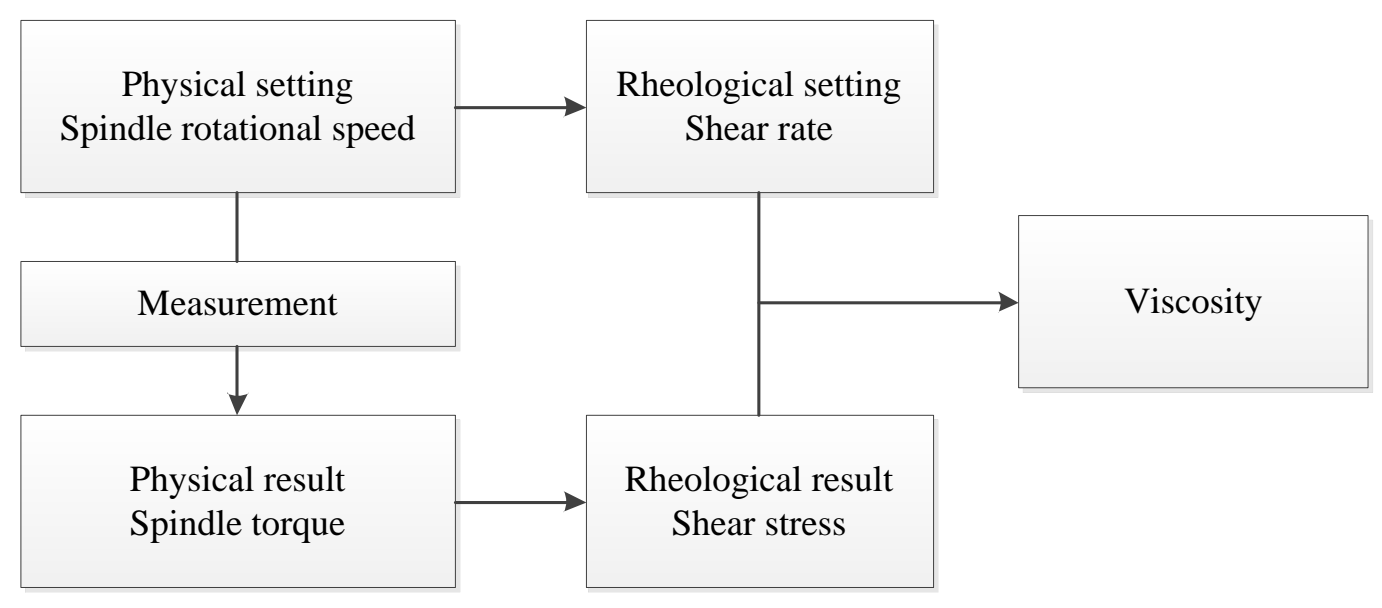

Figure 1. CSR viscosity measurement mode

In the VT mode, a temperature - time profile is preset at a constant spindle speed (i.e. constant shear rate). The spindle torques are measured in relation to various temperatures. The variation of viscosity with temperature was studied in the range from 
30 to $80{ }^{\circ} \mathrm{C}$ with a heating rate of $0.2{ }^{\circ} \mathrm{C} \cdot \mathrm{min}^{-1}$.

Each viscosity measurement was done in triplicate and average results reported.

The fresh bio-oil is unstable, its viscosity changes with time due to carbonyl coupling reactions. The bio-oil samples have been stored for two years. To avoid the effect of the possible aging on the viscosity measurements, the accelerated aging test was performed for the bio-oil samples. In the test, the samples were heated to $80{ }^{\circ} \mathrm{C}$ for $24 \mathrm{~h}$ and their viscosities were measured in the CSR mode after cooling. Detailed information about the accelerated aging test can be found in the literature. ${ }^{17}$ The results have shown that the viscosity values of the bio-oil samples before and after heating kept unchanged, which indicated that the bio-oil samples were totally aged.

\section{MODEL PARAMETER ESTIMATION AND MODEL EVALUATION}

The Arrhenius and WLF models are employed to fit the viscosity-temperature experimental data of four bio-oil samples. Some estimation of the model parameters in the Arrhenius and WLF models was required.

The parameters $\eta_{A}$ and $E_{A}$ in the Arrhenius model are optimised by means of a direct search method for minimising the sum of squared residuals (SSR):

$$
\mathrm{SSR}=\sum_{i=1}^{n_{d}}\left[\eta_{e}\left(T_{i}\right)-\eta_{c}\left(T_{i}\right)\right]^{2}
$$

where $i$ represents the $i$ th data point, $n_{d}$ is the number of data points, $\eta_{e}$ is the experimental data and $\eta_{c}$ is the viscosity value calculated from the model. The SSR values for large grid values $\left(\ln \eta_{A}=-10 \sim-20\right.$ step $0.001 ; E_{A}=40 \sim 60$ step 0.001 $\mathrm{kJ} \cdot \mathrm{mol}^{-1}$ ) were calculated and shown as a 3D scatter plot, where the optimised 
parameter values could be obtained from the lowest bottom point of the plot.

Four parameters $\left(\eta_{\mathrm{wLF}}, C_{1}, C_{2}\right.$ and $\left.T_{r}\right)$ in the WLF model are optimised by using the Levenberg-Marquardt algorithm, an iterative procedure, which has been usually used for solving nonlinear fitting problems. ${ }^{18}$ In this work, the implementation of this algorithm was performed by virtue of DataFit ${ }^{\circledR}$. To start a minimisation, an initial guess for the parameters should be provided. The $C_{1}$ and $C_{2}$ values published in the literature were in the ranges of $5-20$ and $100-200 \mathrm{~K}$, respectively. ${ }^{5}$ Therefore, the initial guess values of $C_{1}$ and $C_{2}$ are set as 12 and $150 \mathrm{~K}$, respectively. In general, $T_{r}$ is in the experimental temperature range. Therefore, the initial guess of $T_{r}$ was set as the median value of the temperature range investigated in this work, i.e., $338 \mathrm{~K}$. The initial guess of $\eta_{\mathrm{wLF}}$ was set as the median value of the viscosity values in the whole temperature range.

Generally, the suitability of any model for fitting experimental data is usually assessed by the coefficient of determination $\left(\mathrm{R}^{2}\right)$. The root mean square deviation, RMSD (Equation (5)), is selected as an additional statistical measures for evaluation of the goodness-of-fit of the model.

$$
\mathrm{RMSD}=\sqrt{\frac{1}{n_{d}} \sum_{i=1}^{n_{d}}\left[\eta_{e}\left(T_{i}\right)-\eta_{c}\left(T_{i}\right)\right]^{2}}
$$

The closer the $\mathrm{R}^{2}$ value approaches 1 and the lower the RMSD value is, the stronger the correlation between the experimental data and the curve predicted by the model. ${ }^{19}$ Also a plot of residuals (i.e. the difference between experimental and predicted data) is used, with the basic assumption that residuals followed a normal distribution with a zero mean. 


\section{RESULTS AND DISCUSSION}

The general properties, such as water content, $\mathrm{pH}$ value, elemental composition and higher heating value (HHV) were measured or calculated to characterise the biooil samples, and the results are summarised in Table 2. Based on the results included in Table 2 the following observations are noted: (1) the higher heating value of the biooil from MXG is lower than that of the bio-oils from beech wood; (2) the water content of the bio-oil from MXG is higher than that of the bio-oils from beech wood; (3) for the bio-oils from beech wood, the carbon content and HHV of the bio-oil increase and the oxygen content of the bio-oil decreases with the increase in pyrolysis temperature (from 450 to $550{ }^{\circ} \mathrm{C}$ ).

Figure 2 shows the viscosity versus shear rate relationships for all bio-oil samples at different temperatures. In these figures, the symbol $\dot{\gamma}$ represents the shear rate. From Figure 2, all experiment results have shown that the viscosity, at various constant temperatures, is almost constant over the entire range of shear rates examined for each bio-oil sample. The results indicate that the viscosity of all bio-oil samples is independent of shear rate and that all bio-oil samples are essentially Newtonian in fluid character. 
Table 2. General properties of bio-oil samples ${ }^{\text {a }}$

\begin{tabular}{|c|c|c|c|c|c|c|c|}
\hline \multirow{2}{*}{ Sample } & \multirow{2}{*}{ Water content / wt. $\%$} & \multirow{2}{*}{$\mathrm{pH}$ value } & \multicolumn{4}{|c|}{ Elemental composition } & \multirow{2}{*}{$\mathrm{HHV} / \mathrm{MJ} \mathrm{kg}^{-}$} \\
\hline & & & C / wt.\% & $\mathrm{H} / \mathrm{wt} . \%$ & $\mathrm{~N} / \mathrm{wt} . \%$ & $\mathrm{O}^{\mathrm{b}} / \mathrm{wt} . \%$ & \\
\hline BW1 & $23.07 \pm 1.23$ & $2.72 \pm 0.02$ & 41.27 & 6.28 & 0.32 & 52.13 & $18.25 \pm 0.76$ \\
\hline BW2 & $20.65 \pm 1.49$ & $2.80 \pm 0.04$ & 42.78 & 5.00 & 0.51 & 51.71 & $18.71 \pm 0.27$ \\
\hline BW3 & $20.94 \pm 1.35$ & $2.62 \pm 0.01$ & 49.04 & 7.39 & 0.51 & 43.06 & $19.06 \pm 0.56$ \\
\hline $\mathrm{MXG}$ & $25.74 \pm 1.87$ & $2.49 \pm 0.01$ & 41.01 & 7.37 & 0.47 & 51.15 & $18.02 \pm 0.39$ \\
\hline
\end{tabular}

${ }^{\mathrm{a}}$ wet basis

${ }^{\mathrm{b}}$ by difference 

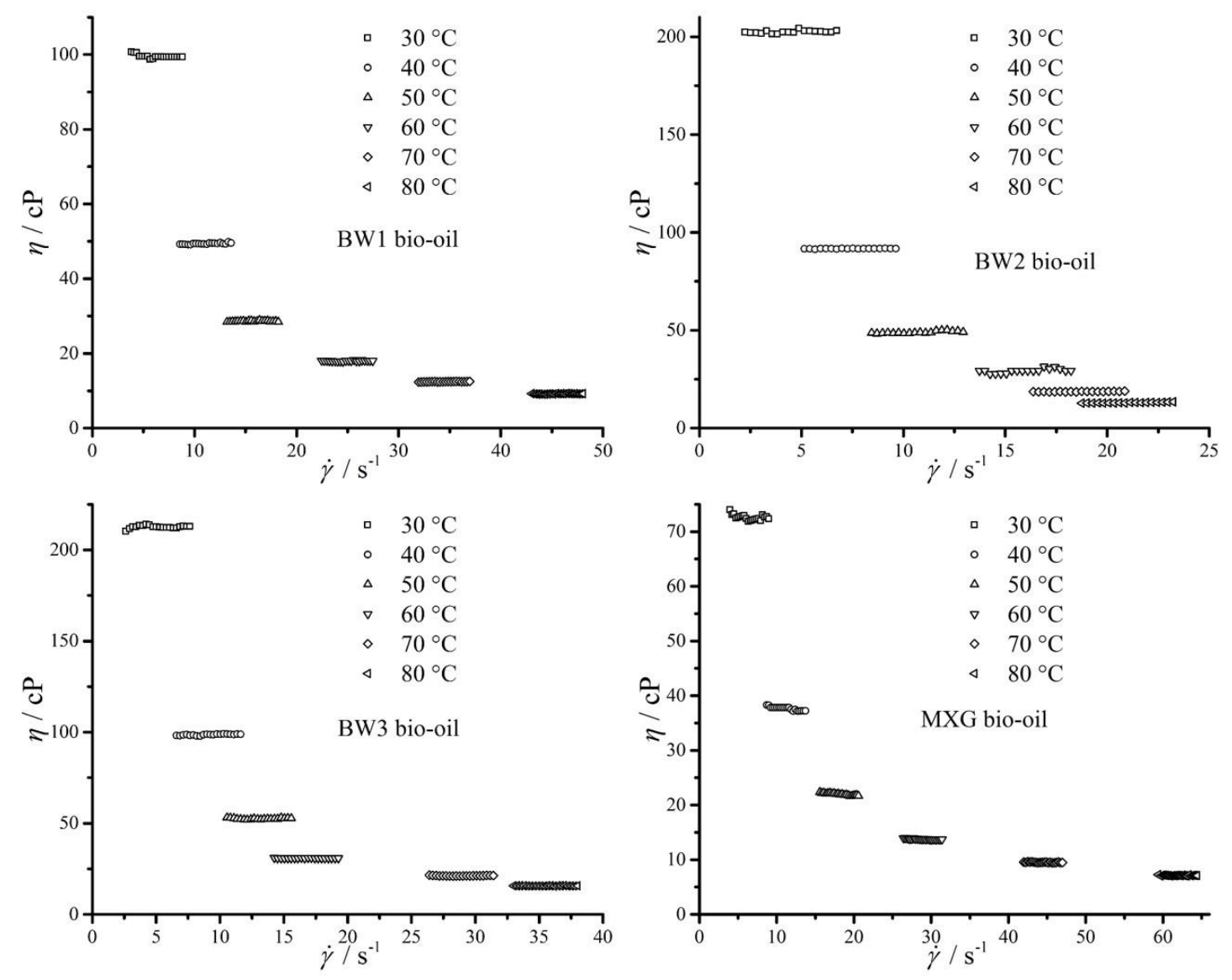

Figure 2. Viscosity versus shear rate relationship at different temperatures

The dependence of viscosity upon temperature for all bio-oil samples is reported in Figure 3. From this figure, the following observations could be noted. (1) As the temperature increases, the viscosity of all bio-oils reduces rapidly, and then at relatively high temperature the viscosity changes slower. By increasing the temperature from 30 to $80{ }^{\circ} \mathrm{C}$, the viscosity of $\mathrm{BW} 1, \mathrm{BW} 2, \mathrm{BW} 3$ and $\mathrm{MXG}$ decreases by $90.7 \%, 93.3 \%$, 92.6\% and 90.2\% respectively. (2) At the same temperature the viscosity of bio-oil from Miscanthus is lower than that of the bio-oils from beech wood. (3) For the bio-oils from beech wood, the higher the pyrolysis temperature the higher the viscosity of bio-oil produced. 
The applied temperature not only provides sufficient energy to break down the internal structure within bio-oil rapidly by reducing attraction forces between molecules, also promotes molecular interactions, ${ }^{20}$ eventually the reduction of internal structure stabilises by increased molecular interactions. In overview, the viscosity of bio-oil decreases as the temperature increases.
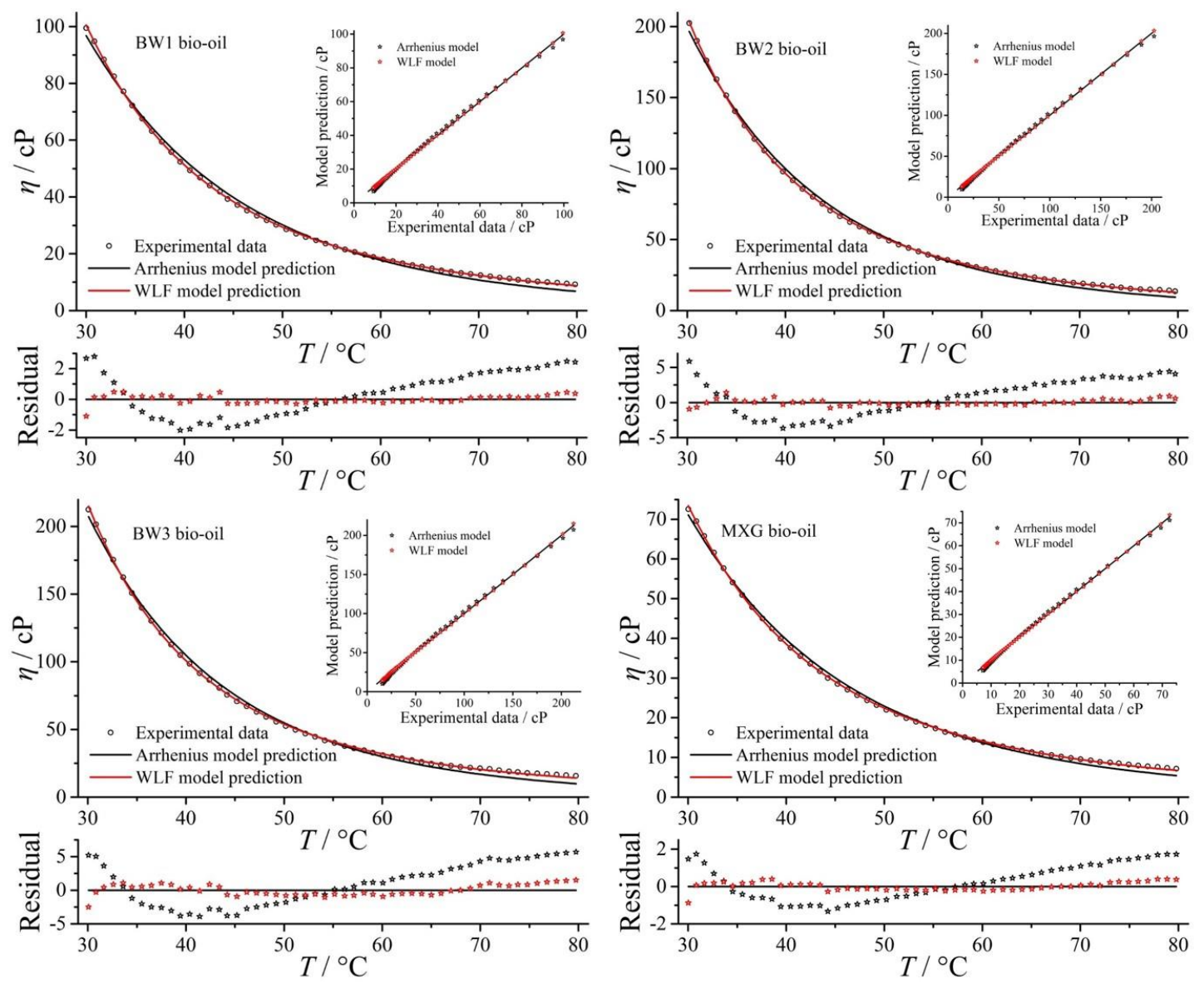

Figure 3. Comparison between the experimental data and the data predicted by the

Arrhenius and WLF models for all bio-oil samples

The Arrhenius and WLF models were used to analyse the viscosity-temperature experimental data of all bio-oil samples. To determine the parameter values of the 
Arrhenius model, the SSR values at various $\ln \left(\eta_{A}\right)$ and $E_{A}$ values for all bio-oil samples were calculated and shown in Figure 4. The optimal values of the Arrhenius model parameters for all bio-oil samples were obtained according to the results demonstrated in Figure 4. The parameters of the WLF model for fitting the viscosity-temperature experimental data were optimised by means of the Levenberg-Marquardt algorithm in the DataFit environment. The statistical calculations for fitting the experimental data to the Arrhenius and WLF models were performed. The model parameters and corresponding statistical results are listed in Table 3 . The comparison between the viscosity-temperature experimental data and the curve predicted by the Arrhenius and WLF models for all bio-oil samples is shown in Figure 3. Based on the results listed in Table 3 and demonstrated in Figure 3, the following observations can be made. First, there was a relatively large discrepancy in the low and high temperature regions of the viscosity-temperature profile for the description of the Arrhenius model. Second, the residuals of the WLF model are effectively close to zero and do not show any pattern, which indicates that the WLF model gives a very good description of the viscosity as a function of the temperature (for all cases, the $\mathrm{R}^{2}$ values given by the WLF model were greater than 0.9998). Third, the WLF model can hold over the entire temperature range investigated. In this aspect, the WLF model is better than the Arrhenius model. This conclusion is similar to the result for a polycarbonate melt reported in the literature. ${ }^{21}$ According to the above analysis results, the WLF model is more suitable than the Arrhenius model for the description of the viscosity-temperature experimental data of bio-oils. 

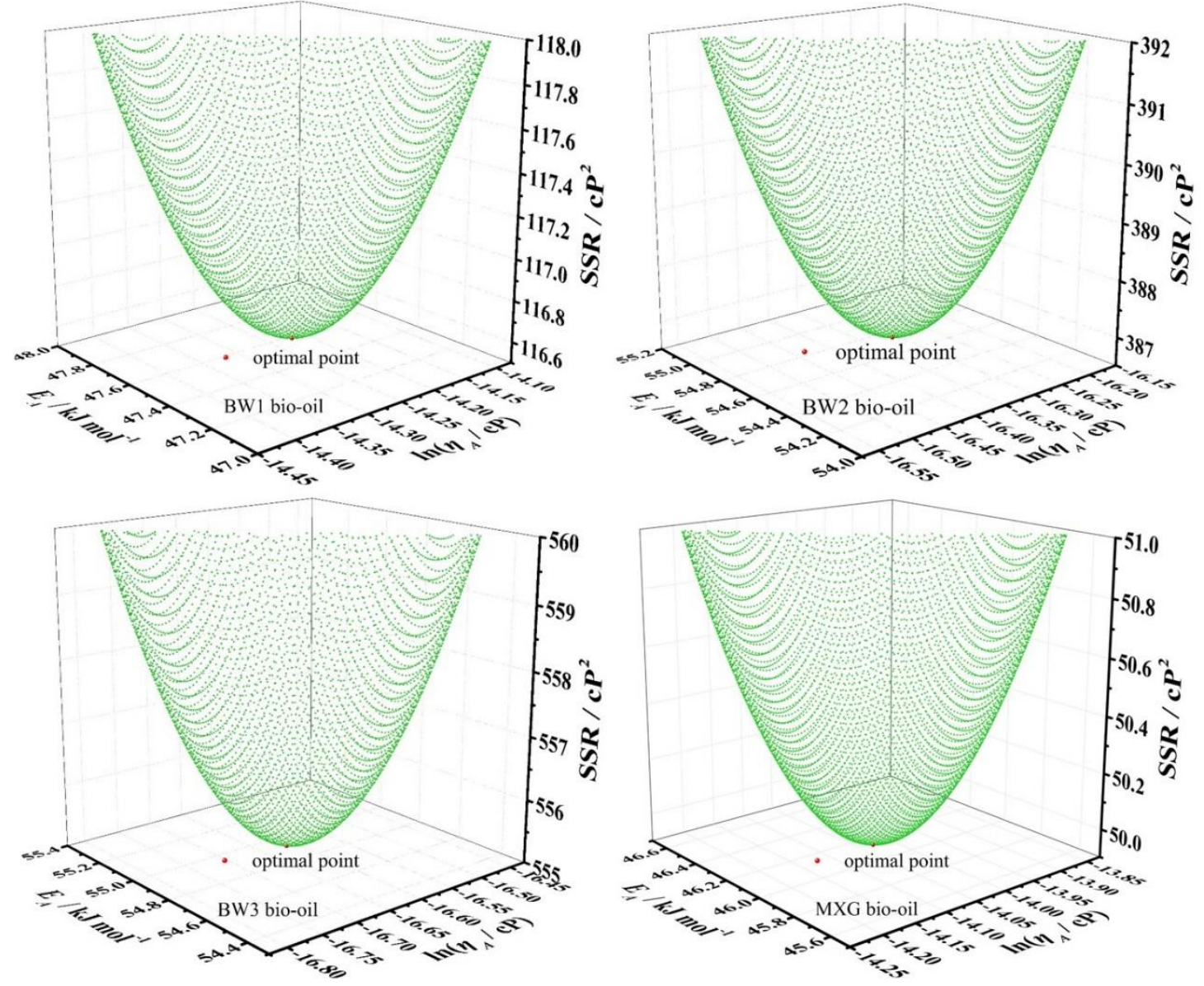

Figure 4. 3D plot of SSR versus $\ln \left(\eta_{A}\right)$ and $E_{A}$ for Arrhenius model 
Table 3. Parameter values of Arrhenius and WLF models together with $\mathrm{R}^{2}$ and SSR values

\begin{tabular}{|c|c|c|c|c|c|c|c|c|c|c|c|}
\hline \multirow{2}{*}{ Bio-oil sample } & \multicolumn{4}{|c|}{ Arrhenius model } & \multicolumn{7}{|c|}{ WLF model } \\
\hline & $\ln \left(\eta_{A} / \mathrm{cP}\right)$ & $E_{A} / \mathrm{kJ} \mathrm{mol}^{-1}$ & $\mathrm{R}^{2}$ & RMSE / cP & $\ln \left(\eta_{\mathrm{wLF}} / \mathrm{cP}\right)$ & $C_{1}$ & $C_{2} / \mathrm{K}$ & $T_{r} / \mathrm{K}$ & $C_{2} / C_{1} / \mathrm{K}$ & $\mathrm{R}^{2}$ & RMSE / cP \\
\hline BW1 & -14.258 & 47.464 & 0.9864 & 1.497 & 3.196 & 6.339 & 118.402 & 326.909 & 18.678 & 0.9999 & 0.266 \\
\hline BW2 & -16.358 & 54.578 & 0.9871 & 2.728 & 3.481 & 6.396 & 126.614 & 331.565 & 19.796 & 0.9999 & 0.445 \\
\hline BW3 & -16.630 & 54.867 & 0.9863 & 3.267 & 3.564 & 6.007 & 127.854 & 332.058 & 21.284 & 0.9998 & 0.825 \\
\hline MXG & -14.032 & 46.120 & 0.9872 & 0.980 & 2.946 & 6.771 & 117.187 & 326.509 & 17.307 & 0.9998 & 0.231 \\
\hline
\end{tabular}


According to the literature, ${ }^{15}$ the higher the $C_{2} / C_{1}$ value of the WLF model is, the stronger the temperature dependence effect of the viscosity is. From the results included in Table 3, the strength of the temperature dependence effect of the viscosity follows the following sequence for all bio-oil samples: BW3 > BW2 > BW1 > MXG.

The applicability of the WLF model for the estimation of the viscosity-temperature relationship of bio-oils in a wider temperature range is our next research work.

\section{CONCLUSIONS}

(1) The viscosity of the BW1, BW2, BW3 and MXG bio-oils is independent on the shear rate. All bio-oil samples considered in this work are Newtonian fluids.

(2) The viscosity of all bio-oils was highly sensitive to temperature. By increasing the temperature from 30 to $80{ }^{\circ} \mathrm{C}$ the viscosity decreases by $90.7 \%, 93.3 \%, 92.6 \%$ and $90.2 \%$.

(3) There is a relatively large discrepancy between the viscosity-temperature experimental data and the data predicted by the Arrhenius model for each bio-oil sample, especially in the low and high temperature regions of the viscosity-temperature profile.

(4) The WLF model gives a very good description of the viscosity as a function of temperature. According to the WLF model parameters, the BW3 bio-oil has the strongest viscosity-temperature dependence, while the MXG bio-oil has the weakest viscosity-temperature dependence.

\section{- ACKNOWLEDGMENTS}


Junmeng Cai would like to acknowledge the financial support from the IRSES ECOFUEL programme (FP7-PEOPLE-2009-IRSES Grant 246772). Scott Banks and Anthony V. Bridgwater would like to acknowledge the collaboration and funding through the EPSRC Grant (NO. EP/K036548/1) “Development of Fast Pyrolysis Based Advanced Biofuel Technologies for Biofuels".

\section{- REFERENCES}

1. Bridgwater, A. V., Review of fast pyrolysis of biomass and product upgrading. Biomass and Bioenergy 2012, 38, 68-94.

2. Yang, Z.; Kumar, A.; Huhnke, R. L., Review of recent developments to improve storage and transportation stability of bio-oil. Renewable and Sustainable Energy Reviews 2015, 50, 859-870.

3. Haj-Kacem, R. B.; Ouerfelli, N.; Herráez, J.; Guettari, M.; Hamda, H.; Dallel, M., Contribution to modeling the viscosity Arrhenius-type equation for some solvents by statistical correlations analysis. Fluid Phase Equilibria 2014, 383, 11-20.

4. Dahlquist, E., Technologies for Converting Biomass to Useful Energy: Combustion, Gasification, Pyrolysis, Torrefaction and Fermentation. CRC Press: 2013.

5. Soto-Castruita, E.; Ramírez-González, P. V.; Martínez-Cortés, U.; QuiñonesCisneros, S. E., Effect of the temperature on the non-Newtonian behavior of heavy oils. Energy \& Fuels 2015, 29, 2883-2889.

6. Brown, R. C.; Stevens, C., Thermochemical Processing of Biomass: Conversion into Fuels, Chemicals and Power. Wiley: 2011. 
7. Seeton, C., Viscosity-temperature correlation for liquids. Tribology Letters 2006, $22,(1), 67-78$.

8. Reynolds, O., On the theory of lubrication and its application to Mr. Beauchamp Tower's experiments, including an experimental determination of the viscosity of olive oil. Proceedings of the Royal Society of London 1886, 40, (242-245), 191-203.

9. Leroy, J.; Choplin, L.; Kaliaguine, S., Rheological characterization of pyrolytic wood derived oils: existence of a compensation effect. Chemical Engineering Communications 1988, 71, (1), 157-176.

10. Thangalazhy-Gopakumar, S.; Adhikari, S.; Ravindran, H.; Gupta, R. B.; Fasina, O.; Tu, M.; Fernando, S. D., Physiochemical properties of bio-oil produced at various temperatures from pine wood using an auger reactor. Bioresource Technology 2010, 101, (21), 8389-8395.

11. Abedi, J.; Nourozieh, H.; Kariznovi, M.; Seyedeyn-Azad, F., Thermo-physical properties of bio-oil and its fractions: Measurement and analysis. The Canadian Journal of Chemical Engineering 2015, 93, (3), 500-509.

12. Raouf, M. A.; Williams, C. R., General rheological properties of fractionated switchgrass bio-oil as a pavement material. Road Materials and Pavement Design 2010, 11, (sup1), 325-353.

13. Williams, M. L.; Landel, R. F.; Ferry, J. D., The temperature dependence of relaxation mechanisms in amorphous polymers and other glass-forming liquids. Journal of the American Chemical Society 1955, 77, (14), 3701-3707.

14. Dobson, G. R., The Dynamic Mechanical Properties of Bitumen. British Petroleum 
Company: 1969.

15. Sopade, P. A.; Halley, P.; Bhandari, B.; D’Arcy, B.; Doebler, C.; Caffin, N., Application of the Williams-Landel-Ferry model to the viscosity-temperature relationship of Australian honeys. Journal of Food Engineering 2002, 56, (1), 67-75.

16. Banks, S. W.; Nowakowski, D. J.; Bridgwater, A. V., Fast pyrolysis processing of surfactant washed Miscanthus. Fuel Processing Technology 2014, 128, (0), 94-103.

17. Meng, J.; Moore, A.; Tilotta, D.; Kelley, S.; Park, S., Toward understanding of biooil aging: Accelerated aging of bio-oil fractions. ACS Sustainable Chemistry \& Engineering 2014, 2, (8), 2011-2018.

18. Rice, J. R., Mathematical Software. Elsevier Science: 2014.

19. Rao, S. S.; Rao, S. S., Engineering Optimization: Theory and Practice. Wiley: 2009.

20. Wan Nik, W.; Eng Giap, S.; Masjuki, H.; Senin, H. In Application of modified power law and Arrhenius relationship in studying rheological behavior of bio-oils, Materials Science Forum, 2006; Trans Tech Publ: 2006; pp 147-152.

21. Lomellini, P., Viscosity - temperature relationships of a polycarbonate melt: Williams - Landel - Ferry versus Arrhenius behaviour. Die Makromolekulare Chemie 1992, 193, (1), 69-79. 